\title{
ROTATION-PULSATION COUPLING IN THE BIPOLAR PREPLANETARY NEBULA, V HYA
}

\author{
MARK MORRIS and CECILIA BARNBAUM \\ Department of Astronomy The University of California at Los Angeles Los Angeles, CA \\ 90024 USA
}

High-resolution optical spectra were taken of the carbon star V Hydrae at 10 different epochs spanning two stellar periods using the Hamilton Echelle Spectrograph at Lick Observatory. Velocities were determined at each epoch by performing a cross-correlation analysis against the spectra of standard stars with previouslydetermined velocities. The velocities of individual atomic absorption and emission lines, and their variations with phase, were also determined. The rising and falling of the photosphere is clearly in evidence, with an amplitude about the mean of $\pm 5 \mathrm{~km} \mathrm{~s}^{-1}$ and a phase consistent with the expectation that maximum light occurs at minimum radius. In addition, the spectra were subjected to a rotational broadening analysis, in which we determined $\mathrm{V} \sin (\mathrm{i})$, where $\mathrm{V}$ is the presumed equatorial rotation velocity, by minimizing the differences between the spectra of $\mathrm{V}$ Hya and a number of artificially broadened comparison stars showing no evidence for broadening. Mechanisms other than stellar rotation, including both turbulence and opacity, were found to be unlikely contributors to the broadening. The very unusual rotation velocity (for a red giant) was found to vary between about 10 and $18 \mathrm{~km} \mathrm{~s}^{-1}$, with an average of $\approx 14 \mathrm{~km} \mathrm{~s}^{-1}$. The relative phase of the rotation velocity curve is consistent with the hypothesis that the pulsation leads to a periodically varying moment of inertia. The assumption of angular momentum conservation, coupled with the radial velocity and $\mathrm{V} \sin (\mathrm{i})$ curves, permits a deduction of the equilibrium stellar radius: The similar magnitudes of the rotation and pulsation velocities imply that they are dynamically coupled, leading to a latitude-dependent circulation which probably affects the mass loss. This interaction should also have a strong effect on the convective structure of the envelope. The angular momentum implied for the V Hya atmosphere leads us to the conclusion that it is a commonenvelope binary, and that it is a precursor of a binary nucleus in a bipolar planetary nebula. 